\title{
Spatial Memory and the Monkey Hippocampus: Not All Space Is Created Equal
}

\author{
Pamela Banta Lavenex and Pierre Lavenex*
}

\begin{abstract}
Studies of the role of the monkey hippocampus in spatial learning and memory, however few, have reliably produced inconsistent results. Whereas the role of the hippocampus in spatial learning and memory has been clearly established in rodents, studies in nonhuman primates have made a variety of claims that range from the involvement of the hippocampus in spatial memory only at relatively longer memory delays, to no role for the hippocampus in spatial memory at all. In contrast, we have shown that selective damage restricted to the hippocampus (CA regions) prevents the learning or use of allocentric, spatial relational representations of the environment in freely behaving adult monkeys tested in an open-field arena. In this commentary, we discuss a unifying framework that explains these apparently discrepant results regarding the role of the monkey hippocampus in spatial learning and memory. We describe clear and strict criteria to interpret the findings from previous studies and guide future investigations of spatial memory in monkeys. Specifically, we affirm that, as in the rodent, the primate hippocampus is critical for spatial relational learning and memory, and in a time-independent manner. We describe how claims to the contrary are the result of experimental designs that fail to recognize, and control for, egocentric (hippocampus-independent) and allocentric (hippocampus-dependent) spatial frames of reference. Finally, we conclude that the available data demonstrate unequivocally that the central role of the hippocampus in allocentric, spatial relational learning and memory is conserved among vertebrates, including nonhuman primates.
\end{abstract}

KEY WORDS: $\quad$ space; episodic; parahippocampal; comparative; semantic

\section{MEMORY AND THE HIPPOCAMPUS}

Since the first report describing the profoundly amnesic patient H.M. (Scoville and Milner, 1957), whose amnesic syndrome resulted from bilateral temporal lobe resection, researchers have worked to define the precise role of the medial temporal lobe structures in learning and memory. Although there is still significant debate about the exact nature of the cognitive processes subserved by these structures (Brown and Aggleton, 2001; Eichenbaum et al., 2007; Morris, 2007; Squire et al., 2007;

Laboratory of Brain and Cognitive Development, Department of Medicine, Unit of Physiology, University of Fribourg, FR 1700, Switzerland Grant sponsor: Swiss National Science Foundation; Grant number: PP00A-106701.

*Correspondence to: Pierre Lavenex, Department of Medicine, Unit of Physiology, University of Fribourg, Chemin du Musée 5, 1700 Fribourg, Switzerland. E-mail: pierre.lavenex@unifr.ch
Bird and Burgess, 2008), a prominent theory advocates that the hippocampal formation (comprising the dentate gyrus, hippocampus, subiculum, presubiculum, parasubiculum, and entorhinal cortex) (Lavenex and Amaral, 2000; Amaral and Lavenex, 2007) is essential for the processing of relational information (Cohen and Eichenbaum, 1993; Eichenbaum, 1999; Buckmaster et al., 2004).

One aspect of hippocampal function that has been studied extensively in rodents is its role in spatial relational learning and memory, that is, the formation and use of allocentric representations of space (O'Keefe and Nadel, 1978; Nadel and Hardt, 2004). Indeed, numerous studies have linked the integrity of the hippocampus to a rodent's ability to learn and remember a particular location in the absence of local cues marking that location, that is, in relation to distant environmental cues (Morris et al., 1982; Nadel, 1991; Schenk et al., 1995). Electrophysiological studies have further implicated the rodent hippocampus in spatial learning and memory (O'Keefe and Dostrovsky, 1971; O’Keefe, 2007).

\section{SPATIAL MEMORY IN MONKEYS}

Given our extensive understanding of the role of the hippocampal formation in spatial relational learning and memory in the rodent, and our obvious desire and need to understand the role of the human hippocampus in learning and memory for clinical reasons, it is clear that nonhuman primates are a crucial group in which experimental analyses of hippocampal function must be thoroughly investigated. Nevertheless, comparable studies of the role of the monkey hippocampus in spatial learning and memory are few, and their findings often contradictory. For example, whereas some experiments report that hippocampallesioned monkeys exhibit impaired performance in various spatial memory tasks (Parkinson et al., 1988; Angeli et al., 1993; Beason-Held et al., 1999; Alvarado et al., 2002; Hampton et al., 2004; Banta Lavenex et al., 2006; Bachevalier and Nemanic, 2008), other experiments have failed to show any spatial memory impairments at all (Murray and Mishkin, 
1998; Malkova and Mishkin, 2003; Hampton et al., 2004; Bachevalier and Nemanic, 2008). These lesion studies stand in contrast to electrophysiological studies that support the hypothesis that the monkey hippocampus is involved in spatial learning and memory (Rolls et al., 1989; Feigenbaum and Rolls, 1991; Ono et al., 1991; Rolls and O’Mara, 1995; GeorgesFrancois et al., 1999; Hori et al., 2003; Rolls and Xiang, 2006), as well as functional magnetic resonance imaging and virtual reality experiments that similarly implicate the human hippocampal formation in spatial learning and memory (e.g., Bird and Burgess, 2008).

Indeed, the many incongruities in the literature might lead to the conclusion that the primate hippocampus functions in a manner fundamentally different from that of the rodent, a hypothesis that has drastic consequences for our understanding of the function of the human hippocampal formation. Although this conclusion is neither very satisfying nor parsimonious, there is, to date, no coherent interpretation of the discrepant findings reported in the literature. Recently, however, we have conducted a series of experiments using a novel experimental paradigm designed to assess the role of the monkey hippocampal formation in spatial relational learning and memory (Banta Lavenex et al., 2006; Lavenex and Banta Lavenex, 2006; Lavenex et al., 2007b). Our experiments have shown that the primate hippocampus is unequivocally involved in the processing of allocentric, spatial relational information, thus confirming its conserved function across mammalian species (Banta Lavenex et al., 2006). In this commentary, we discuss a unifying framework that explains the apparently discrepant results regarding the role of the monkey hippocampus in spatial learning and memory. We describe clear and strict criteria to interpret the findings from previous studies and guide future investigations of spatial memory in monkeys.

\section{DISTINCT SPATIAL FRAMES OF REFERENCE}

In their seminal work "The hippocampus as a cognitive map," O'Keefe and Nadel (1978) elucidate the arguments in favor of the existence of two separate and distinct spatial systems: (1) An egocentric, or viewpoint-dependent, representation of space defined in a frame of reference centered on a subject's own body position; and (2) an allocentric, or viewpoint-independent, representation of space defined in a frame of reference centered on the objects constituting the environment, and therefore independent from the subject's own location or orientation. It has been previously demonstrated in rodents, and is now well-accepted, that allocentric behavioral strategies are critically dependent on the integrity of the hippocampus (Morris et al., 1982; Nadel, 1991; Schenk et al., 1995; Morris, 2007). In contrast, egocentric behavioral strategies are not impacted by hippocampal damage (Nadel, 1991; Schenk et al., 1995; Nadel and Hardt, 2004). As we will describe below, critical evaluation of the tasks used previously by other researchers reveals that the discrepancies in the monkey hippocampus literature can be clearly and consistently explained as a failure to recognize and control for the fact that monkeys have normally access to both egocentric (hippocampus-independent) and allocentric (hippocampus-dependent) representations of space (Nadel and Hardt, 2004). Experiments separating these two distinct spatial frames of reference, however, are absolutely critical in order to decipher the exact role of the primate medial temporal lobe structures in spatial learning and memory (Banta Lavenex et al., 2006).

In this commentary, we review the findings of select studies that have investigated the role of the monkey medial temporal lobe structures in spatial learning and memory. Specifically, we describe in detail four recently employed behavioral paradigms, and explain how particular flaws in the conceptual design of these studies have led to inconsistencies in their results when taken together, as well as to flawed interpretations as to the role of the monkey hippocampus in spatial learning and memory. We also describe our experimental paradigm that has yielded experimental results consistent with a large body of well-established work in rodents. Finally, we identify the specific factors that are critical to recognize and incorporate into any experiment, aiming to define the role of particular brain structures in spatial learning and memory.

\section{THE DELAYED-NONMATCHING-TO-LOCATION PARADIGM}

We begin with a description of the paradigm that is most often used to test spatial memory in monkeys, namely, the delayed-nonmatching-to-location (DNML) task performed in the Wisconsin General Testing Apparatus (WGTA). Figure 1 illustrates the experimental apparatus, which typically employs a tray containing three wells that is positioned in front of a cage in which the monkey is placed during testing. In the standard version of the task, during what is referred to as the sample phase, the monkey is first shown one object that is placed in one well location (Fig. 1A). In the test phase, after a defined delay, the monkey is shown two identical objects: one in the previous location, and the other in a new location. As this task rewards the novel location (DNML), the monkey will find a reward by choosing (i.e., lifting) the object in the novel location. However, in order to choose the novel location, the monkey must be able to remember where the object was located during the sample phase (Fig. 1A).

A number of studies have employed the DNML task as a general assay for hippocampal function (e.g., Parkinson et al., 1988; Angeli et al., 1993; Murray and Mishkin, 1998; Malkova and Mishkin, 2003). Researchers have reasoned that this is a spatial task, since the monkey is required to remember and identify the location where an object was previously seen. Researchers further reasoned that since the hippocampal formation has been shown to be responsible for processing spatial information in rodents, monkeys with hippocampal lesions should be impaired at performing the DNML task. As reasonable as this logic may seem, studies using the DNML paradigm 
A
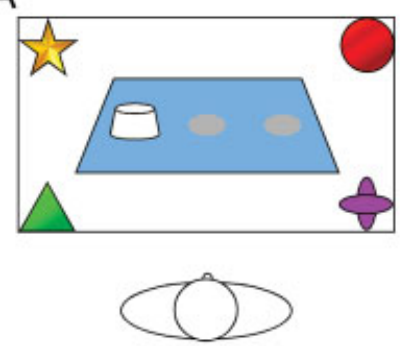

B

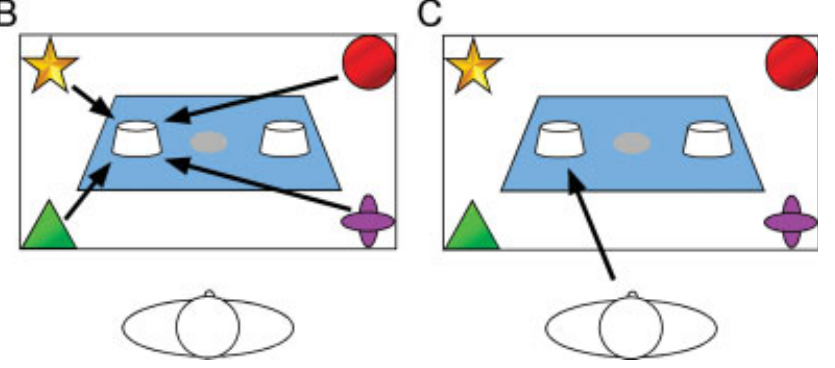

FIGURE 1. Schematic representation of the delayed-nonmatching-to-location (DNML) task performed in the Wisconsion General Testing Apparatus (WGTA). (A) Sample phase: an object is placed in one-well location of a three-well tray positioned in front of the cage in which the monkey is placed during testing. (B) After a defined delay, the subject must choose the object that has been placed in the novel location to obtain a food reward. The location of the object presented during the sample phase can be encoded based on its relation to other objects constituting the environment. This corresponds to an allocentric coding of space. (C) The location of the object presented during the sample phase can also be encoded based on its position relative to the subject's own body position, which in this particular case is on the left. This corresponds to an egocentric coding of space. [Color figure can be viewed in the online issue, which is available at www.interscience. wiley.com.]

have failed to show decreased performance of monkeys with hippocampal lesions as compared to controls [(Murray and Mishkin, 1998); using a 10-well tray, but with only two, rather than three, potential locations during sample and test trials], whereas "extra-hippocampal" lesions impaired performance (Parkinson et al., 1988; Angeli et al., 1993). How can these experimental results be explained?

In experiments employing the DNML task as described above, it is clear that researchers have neglected two critical points. First, both allocentric and egocentric spatial frames of reference can be used to encode and remember the location of a particular object placed on the tray: As illustrated in Figure 1 , a normal subject can remember the location of the object based on its relative position defined in relation to other objects constituting the environment (Fig. 1B), that is, in an allocentric spatial frame of reference. However, a normal subject can also remember the location of the object based on its position relative to its own body position, for example, on the left, in the center, or on the right (Fig. 1C), that is in an egocentric spatial frame of reference. Second, as previously described, rodent studies have shown that egocentric strategies are not dependent on the integrity of the hippocampus.
Considering these two points, it is completely understandable, if not predictable, that the DNML task fails to distinguish control monkeys from those with hippocampal lesions. Indeed, the experiment by Murray and Mishkin (1998), which tested monkeys that had received lesions confined to the amygdala and hippocampal formation found preserved performance on the DNML task. In contrast, two studies which examined DNML performance in monkeys that had received "extra-hippocampal" lesions that included the parahippocampal cortex found impaired performance (Parkinson et al., 1988; Angeli et al., 1993). Taken together, these experiments thus argue strongly that the DNML task performed in a WGTA can be solved by using a solely egocentric frame of reference; and lesions of the parahippocampal cortex, and not lesions of the hippocampus, are responsible for impairing performance on tasks such as the DNML that rely on an egocentric coding of space.

In a modified version of the DNML task (Fig. 2), Malkova and Mishkin (2003) evaluated one-trial memory for objectplace associations after either lesion of the hippocampus or the posterior parahippocampal region in the monkey. The task proceeded in the following manner: During the sample phase, two different objects were placed over two different wells of a threewell tray (Figs. 2A,B). In the test phase, after a 6-s delay, one of the two sample objects, together with its exact duplicate,
A

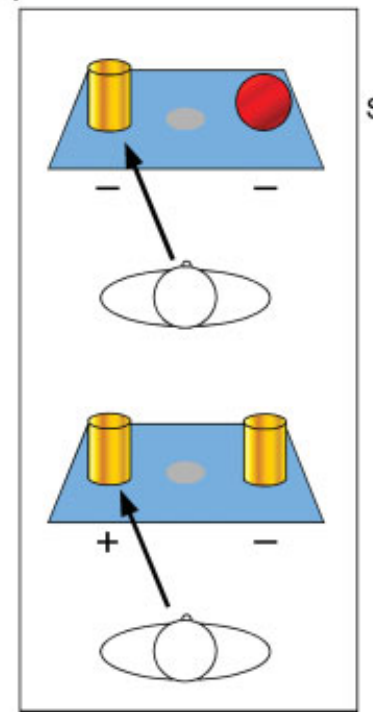

B

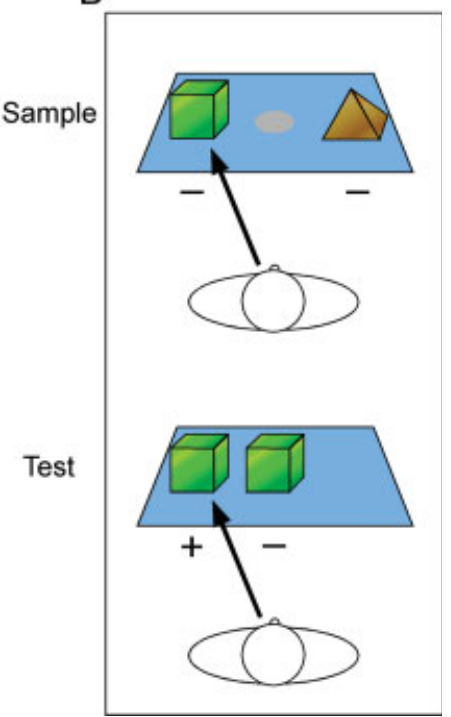

FIGURE 2. Schematic representation of the tasks used by Malkova and Mishkin (2003). An unlike pair of unrewarded objects is presented in the sample phase. In the test phase, one of the two sample objects together with its exact duplicate are presented either over the same two foodwells that were used in the sample phase (A: Object-place trials), or over one of the foodwells used during the sample phase and over the third, previously unused foodwell (B: Place trials). In both conditions, however, the location of the objects presented during the sample phase can be encoded and remembered in an egocentric spatial frame of reference. Thus, in both the object-place and the place trials, the memory of a simple association (i.e., cylinder-left, or block-left, respectively) is sufficient to solve the task. [Color figure can be viewed in the online issue, which is available at www.interscience.wiley.com.] 
were presented either over the same two wells that were used during the sample phase (object-place trials; Fig. 2A), or over one of the wells used during the sample phase and over the third, previously unused well (place trials; Fig. 2B). Twelve of each type of trial were intermixed within a daily session, with an intertrial interval of $15 \mathrm{~s}$. The authors reasoned that the monkeys could solve the object-place trials only by associating each sample object with its location, whereas monkeys could solve the place trials simply by remembering the locations of the two sample objects irrespective of the identity of the object. With respect to this experiment, it is critical to recognize that the spatial location of these objects can be encoded in an entirely egocentric spatial frame of reference. For both the object-place and the place trials, monkeys need only to encode and remember the location of an object that was seen just $6 \mathrm{~s}$ before as defined in relation to their own body position, that is, on the left, at the center, or on the right. Moreover, in the test phase of the place trials, monkeys can even ignore the identity of the object and simply select whatever location had an object in the sample phase (or alternatively, by remembering the location of the uncovered well during the sample phase and then avoiding it during the test phase). Again, however, this can be done using a uniquely egocentric frame of reference. Thus, in both the object-place and the place trials, the memory of a simple association (i.e., cylinder-left or block-left, respectively) is sufficient to solve the task. Consequently, monkeys do not need to rely on an allocentric, spatial relational representation of the environment to solve the task, and even monkeys with selective lesion of brain areas involved in the elaboration of such representations (i.e., the hippocampus) should be able to perform the task. In contrast, monkeys with lesions of the cortical structures involved in the egocentric coding of space (i.e., the parahippocampal cortex) should not be able to perform the task. Not surprisingly, this is precisely what Malkova and Mishkin's experiment has shown: Selective hippocampal lesions had no effect on either task, whereas lesions of the parahippocampal cortex impacted both types of trials (Malkova and Mishkin, 2003). These findings are also consistent with the findings described above from these same researchers reporting the absence of spatial memory deficits in the DNML task following selective hippocampal lesions (Murray and Mishkin, 1998), but strong impairments following lesions that included the hippocampus and the parahippocampal cortex (Parkinson et al., 1988; Angeli et al., 1993).

In another study, Alvarado et al. (2002) evaluated spatial memory performance in a WGTA with a testing tray consisting of nine wells arranged in rows of three (Fig. 3A). Plaques were used to cover one of the nine wells during the sample phase, and two of the nine wells (the previously sampled but now unrewarded well, and a newly rewarded well) during the test phase. As we would predict, based on the fact that animals had access to both egocentric and allocentric spatial frames of reference to solve the task, performance of hippocampal-lesioned monkeys (assessed across several delays from 30 to 600 s) was well above chance level in all testing conditions. However, despite the fact that an ANOVA failed to demonstrate an interac-
A

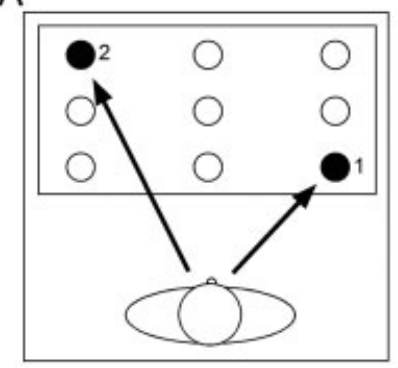

B

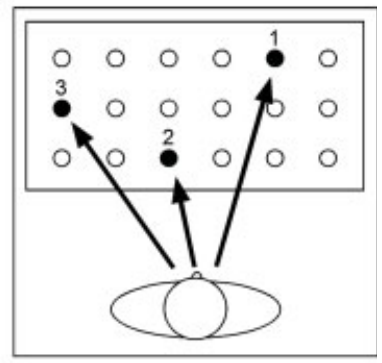

C

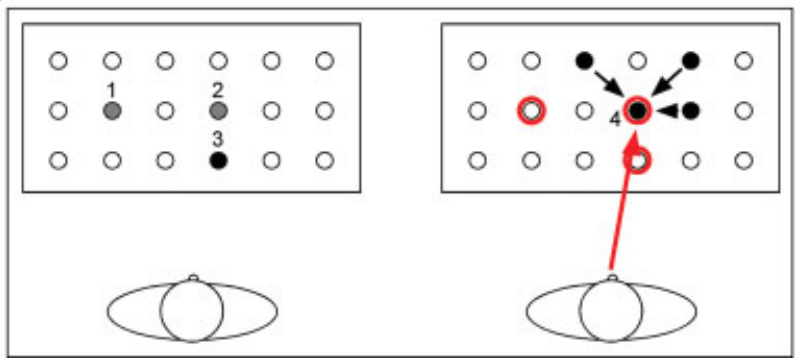

FIGURE 3. Schematic representation of three different tasks used to test spatial memory in a WGTA with a large number of wells. (A) Experimental design used by Alvarado et al. (2002) for a delayed-nonmatching-to-location (DNML) task. The testing tray $(30 \times 63 \mathrm{~cm})$ consisted of nine wells arranged in rows of three (10-cm apart, center to center). (B) Experimental design used by Beason-Held et al. (1999) for a spatial delayed recognition span (DRS) task. The exact dimensions of the design are not reported. (C) Experimental design used by Buckmaster et al. (2004) to evaluate the monkey's reliance on different "spatial relational frames of references" in the performance of a spatial DRS task. The stimulus-tray measured $26.5 \times 68.5 \mathrm{~cm}$ and contained $3 \times 6=18$ possible locations (with a 8.5- and 8-cm spacing in the vertical and horizontal dimensions, respectively). The figure on the left represents a standard trial, during which the monkey is rewarded for choosing a sweet cookie (black) presented in a novel location, while identical, but bitter cookies (gray) are placed at previously rewarded locations. The figure on the right represents a probe trial during which two different spatial frames of references are put into conflict. Again, black circles represent sweet cookies. If the monkey uses a "local" spatial strategy, then the relative spatial location of the cookie designated by the black arrows, as defined in relation to the other previously rewarded locations, predicts that it should be chosen by the monkey since it is a novel, previously unrewarded location. However, if the monkey uses a "global" strategy, then the spatial location of the cookie designated by the red arrow, as defined in relation to the global testing environment that includes both egocentric and allocentric frames of references, predicts that this position should be avoided by the monkey since it is a previously rewarded location. See main text for a detailed description of the procedures. [Color figure can be viewed in the online issue, which is available at www.interscience.wiley.com.]

tion between groups and delays, the published data indicate that the difference between controls and hippocampal-lesioned monkeys was greater at longer delays (group differences: $30 \mathrm{~s}$ : $\mathrm{t}(6)=3.02, P=0.0233 ; 60 \mathrm{~s}: \mathrm{t}(6)=2.1, P=0.0735$; [average 30-60 s delays: $\mathrm{t}(6)=2.73, P=0.0342] ; 120$ s: $\mathrm{t}(6)=$ 3.16, $P=0.0195 ; 600 \mathrm{~s}: \mathrm{t}(6)=4.23, P=0.0055$; [average 120-600 s delays: $\mathrm{t}(6)=6.41, P=0.0007])$. These findings 
highlight two important points with respect to the DNML task: First, if an egocentric representation of space can be used to solve the task, hippocampal-lesioned monkeys will be able to perform above chance level, even though their performance might be different from that of controls who can benefit from the additional information provided by an allocentric, spatial relational representation. Second, whereas an egocentric representation of space can sustain performance over short delays, it is less reliable over longer delays. This issue will be further discussed in the context of another series of experiments.

In sum, it is clear that experiments employing the DNML task in the WGTA, which fail to control for the fact that monkeys have access to both egocentric and allocentric strategies in order to solve the task, will produce results that are contradictory and difficult, if not impossible, to interpret (Belcher et al., 2006).

\section{THE VISUAL-PAIRED COMPARISON PARADIGM}

Another experimental paradigm used to investigate the specific contribution of the medial temporal lobe structures in spatial memory is the visual-paired comparison (VPC) task (Bachevalier and Nemanic, 2008). The chief difference between the VPC and the DNML tasks is the behavioral measure used to infer the cognitive and memory processes evaluated by the two different tasks. In the DNML task, for example, monkeys are required to choose, by grasping, one of several objects in order to obtain a reward. By analyzing the explicit choices of control versus experimentally lesioned subjects, researchers can infer the role of particular brain structures in performing the task. In the VPC task, in contrast, monkeys are not required to respond in an explicit manner (i.e., by grasping), but rather their visual scanning pattern and preferential looking time (i.e., time spent looking at one stimulus or location vs. another) are considered by the experimenter to infer the information that control versus experimentally lesioned monkeys are attending to and remembering, and thus the brain structures implicated in performing the task.

Nevertheless, the same limitations that apply to the DNML task with regard to testing spatial memory, specifically the monkey's ability to rely on allocentric and egocentric information to solve the task, also apply to the VPC task. Bachevalier and Nemanic (2008) tested monkeys in two variations of the VPC task: the spatial-location condition (Fig. 4A) and the object-in-place condition (Fig. 4B). In the spatial-location condition, an image is presented on a screen in front of the subject during the sample phase. In the test phase, two images identical to that presented in the sample phase are shown on the screen, one at the same location and one at a novel location. If the monkey remembers the location of the image presented in the sample phase, it should spend more time looking at the same image presented in the novel location. However, similar to the DNML, the location of the sample image on the screen can be encoded and remembered using both egocentric (e.g., on the right, center, or left of the monkey) as well as allocentric (i.e., in relation to other objects constituting the environment) spa-
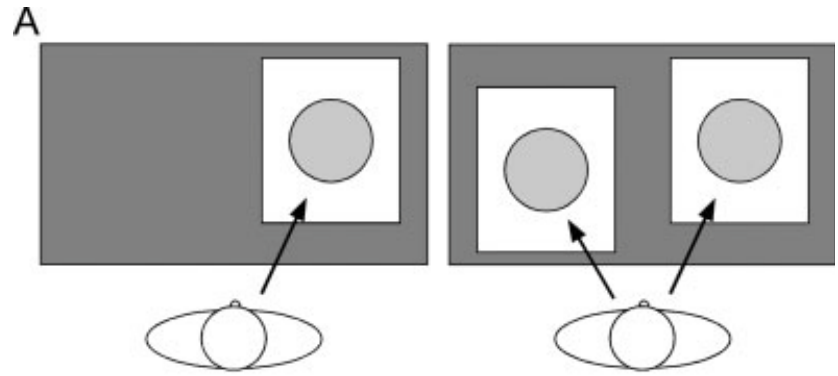

B
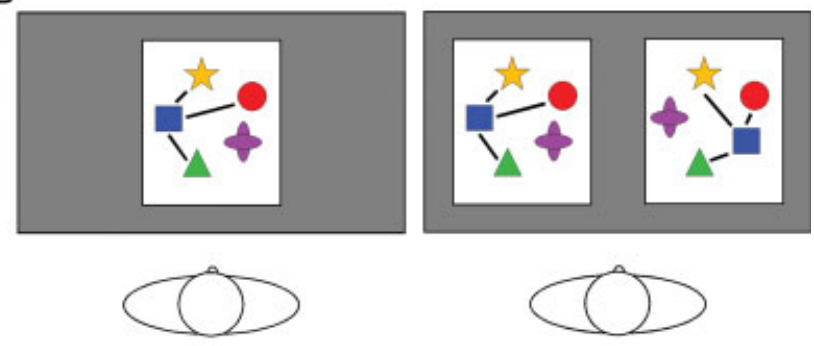

C
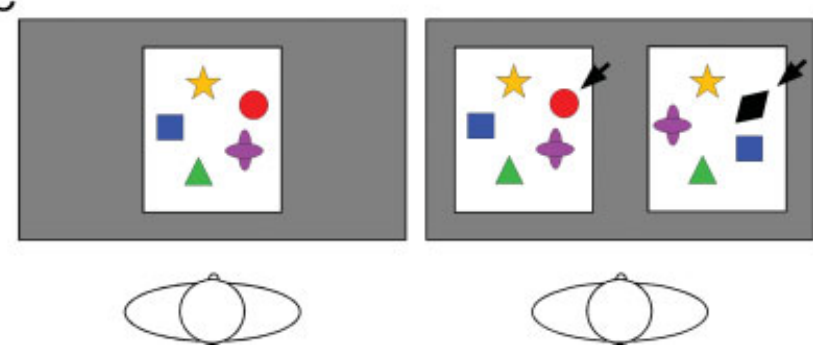

FIGURE 4. Schematic representation of the visual-paired-comparison (VPC) tasks used by Bachevalier and Nemanic (2008). (A) Spatial-location condition: an image is presented on a screen in front of the subject during the sample phase. In the test phase, two images identical to that presented previously are shown on the screen, one at the same location as in the sample phase, and one at a novel location. (B) Object-in-place condition: an image containing a group of five objects is presented in the center of the screen during the sample phase. In the test phase, the same array and a novel array with the same objects differently arranged are presented simultaneously on the left and right side of the screen, respectively. (C) Control condition: an image containing a group of five objects is presented in the center of the screen during the sample phase. In the test phase, the same array and a novel array with one of the objects replaced are presented simultaneously on the left and right side of the screen, respectively. [Color figure can be viewed in the online issue, which is available at www. interscience.wiley.com.]

tial frames of reference. It is therefore not surprising that hippocampal-lesioned monkeys spent the same percentage of time looking at the image appearing in the novel location as shamoperated controls [i.e., no effect of hippocampal lesion; Fig. 5 of (Bachevalier and Nemanic, 2008)]. In contrast, monkeys with aspiration lesions of parahippocampal areas $\mathrm{TH}$ and TF did not exhibit a preference for the image presented in the novel location. Again, these findings provide further corroborating evidence that the hippocampus is necessary for the allocentric, relational coding of space, whereas the parahippocampal cortex is necessary for the egocentric coding of space. 
In the object-in-place condition (Fig. 4B), monkeys are first shown a group of five objects that are presented over a white rectangular background centered on the screen (the sample array). For the test phase, the monkeys are simultaneously presented the sample array alongside a novel array that consists of the same five objects, but with the location of those familiar objects within the array rearranged. As before, if monkeys are able to distinguish between the sample and rearranged arrays, they should spend more time looking at the novel (rearranged) array. First, it is important to note that an egocentric spatial frame of reference cannot be used to determine the position of the array of objects presented during the sample phase, because it is first shown in the center of the screen and then either on the left or on the right of the screen during the test phase. Instead, the location of each object within the array must be defined in relation to that of the other objects constituting the array (i.e., its local environment). Indeed, this is the definition of a spatial relational representation, which codes the location of objects in relation to one another and independently from the position of the observer. In this experiment, Bachevalier and Nemanic found that whereas control monkeys looked preferentially at the reorganized array of objects, neither hippocampal-lesioned nor parahippocampal-lesioned monkeys were able to discriminate the novel array [Fig. 6 of (Bachevalier and Nemanic, 2008)]. Importantly, in their control task, in which one of the five objects was replaced by another, but the configuration of the objects relative to one another remained the same (Fig. 4C), hippocampal-lesioned monkeys exhibited the same preference for the array containing the new object as control monkeys [Fig. 7 of (Bachevalier and Nemanic, 2008)]. These findings confirm that neither simple visual discrimination nor object memory is impaired in hippocampal- or parahippocampal-lesioned monkeys.

Bachevalier and Nemanic considered their results on the object-in-place condition as inconsistent with those reported by Mishkin and colleagues in the object-location condition of the DNML (described above, which hippocampal-lesioned monkeys were able to solve). In an attempt to reconcile these two findings, Bachevalier and Nemanic (2008) reasoned that the fact that the DNML is a problem-solving task, requiring explicit responses, and the VPC an incidental task, requiring no explicit response, might explain the differences. In contrast, we contend that although such differences might contribute to a differential sensitivity of the tasks, the real difference between these two tasks is their specificity. The spatial representations under investigation in the two tasks are fundamentally different, and the differential effect of hippocampal lesions can serve to distinguish the two paradigms. In the DNML studies cited above, the location of the different objects (maximum of three) can be encoded in an egocentric spatial frame of reference (left, center, or right; Figs. 1 and 2), similar to the spatial location task as implemented in the VPC paradigm (left or right; Fig. $4 \mathrm{~A})$. In contrast, in the object-in-place condition of the VPC task (Fig. 4B), the relative position of five different objects must be encoded and remembered, a process that is clearly relational in nature. It is therefore not surprising to observe that, in this condition, hippocampal-lesioned monkeys failed to exhibit a preference to look at the array containing the displaced object(s).

This experiment further highlights another critical point regarding the cognitive processes that subserve egocentric and allocentric spatial coding. Because the elaboration of an allocentric spatial relational representation requires, in part, the integration of egocentric spatial information processed by the parahippocampal cortex, allocentric representations are not possible without egocentric input. This is supported by the finding that monkeys with parahippocampal cortex lesion are also impaired in the object-in-place condition of the VPC paradigm. In contrast, all current lesion studies indicate that egocentric representations persist even in the absence of allocentric processing (i.e., following selective hippocampal lesion). This hypothesis is supported by the fact that monkeys with hippocampal lesions were able to solve the spatial location condition of Bachevalier and Nemanic's VPC paradigm, as well as Mishkin and colleagues' object-location condition in the DNML paradigm.

\section{THE DELAYED RECOGNITION SPAN TASK PARADIGM}

A third experimental paradigm used to investigate the role of the monkey hippocampus in spatial memory is the delayed recognition span (DRS) task performed in a modified WGTA with an 18-well tray (Fig. 3B; Beason-Held et al., 1999). In the sample phase of this task, the monkey is presented with a tray on which a disk covers one well. The monkey must displace the disk in order to obtain a food reward. In the test phase, after a 10-s interval, the monkey is presented with the tray again, but this time there are two disks: one in the original location, and a second disk in a novel location. The monkey is required to displace the disk in the novel location in order to obtain the food reward. Disks are added at novel rewarded locations until the animal makes an error. The number of correct locations chosen before the error is committed constitutes the recognition span score. Monkeys performed 10 trials per day: Eight of the recognition spans were trial unique and two of the recognition spans were fixed sequences repeated twice each day. When unique sequences were presented, hippocampal-lesioned monkeys averaged 1.94 locations before they made an error, whereas control monkeys averaged 2.42 locations. When the repeated sequences were presented, hippocampallesioned monkeys averaged 2.14 locations, whereas control monkeys averaged 3.42 locations. Thus, although control monkeys exhibited surprisingly poor performance on this DRS task, hippocampal-lesioned monkeys still performed significantly worse.

When looking at the 18-well tray it is easy, if not natural, to divide the tray into three distinct parts: left, center, and right. If this is the case, then a simple egocentric spatial representation can be used to solve the task for up to three locations. 
Indeed, the hippocampal-lesioned animals in their study remembered an average of 1.94 and 2.14 locations (on unique and repeated recognition spans, respectively). Whereas control monkeys might have benefited from an allocentric spatial relational representation of the disk locations to encode more reliably previously rewarded locations, hippocampal-lesioned monkeys were still able to access an egocentric representation of space to remember in which general area of the tray they had previously found rewarded locations, and therefore identify which disk covered the novel, rewarded location. Control monkeys also benefited more from the repetition of the sequences and improved their performance by $41 \%$ (as compared to that observed with unique sequences), whereas hippocampallesioned monkeys' performance was only $10 \%$ higher with repeated sequences. The repetition of the same sequence might have benefited control monkeys in their elaboration of a spatial representation encoding the locations of the disks in relation to one another.

As in the previously discussed studies, the main limitation of this DRS task is the fact that both egocentric and allocentric spatial representations could enable animals to solve the task, and thus uncontrolled parameters of the task lead to a differential performance between hippocampal-lesioned and control monkeys. It is also interesting to note that the overall low performance exhibited by both groups of monkeys in this DRS study could have been the result of the monkeys' natural tendency to rely on a win-stay strategy and therefore go back to previously rewarded locations (Lavenex and Banta Lavenex, 2006), instead of choosing the novel rewarded location.

Finally, it is worth discussing a study by Buckmaster et al. (2004) that used a modified version of the DRS task (Fig. 3C) to evaluate the monkeys' reliance on what these authors referred to as different "spatial relational frames of reference." On the fourth location of a four-item DRS task, probe trials were introduced that imposed a conflict between the relative spatial relationships of the previously rewarded locations and their location in relation to the distal environmental cues that comprised the global testing environment (i.e., the array of previously rewarded locations was moved to a different position on the board). Experimental results showed that when faced with this incoherency, control monkeys' performance fell to chance, suggesting that they were encoding both local relational information (i.e., about the relative position of the previously rewarded locations with respect to one another), as well as information defining these locations in relation to the global testing environment (i.e., the room). However, what the authors failed to recognize was that because monkeys always approached the tray from the same direction, the previously rewarded locations could be defined within the testing room using both allocentric as well as egocentric spatial representations. Therefore, because the task failed to preclude the monkeys' use of egocentric information, it cannot be considered to have demonstrated their reliance on allocentric, spatial relational representations of the environment to guide their choices. Indeed, the fact that the monkeys reacted to the incoherency between different sources of spatial information does not indicate that they relied on any one particular source in order to guide their choices in an explicit manner (e.g., Lavenex and Schenk, 1996).

\section{THE OPEN-FIELD DELAYED-MATCHING-TO- LOCATION PARADIGM}

A fourth experimental paradigm designed to evaluate the role of the monkey hippocampus in spatial learning and memory was conceived by Hampton et al. (2004). This task is different from the three previously described experimental designs, because tethered monkeys were allowed to move about in an open-field environment $(4 \times 5 \mathrm{~m})$ in order to complete a match-to-location task (Fig. 5). Moreover, in some of their experiments the authors made an explicit attempt to control for the different spatial frames of reference available to the monkeys performing the tasks, as described below.

In a first experiment, monkeys performed a delayed-matching-to-location task, always starting from the same location in the open-field environment. The task employed four identical potentially rewarded locations (inverted flower pots), with one trial-unique location rewarded on each trial. During the sample phase, monkeys were allowed to search among the four foraging sites until they located the hidden food. After finding the rewarded site, monkeys were allowed to take only a portion of the food before being pulled back to the start location (note that monkeys were not allowed to search any of the other potentially rewarded locations once they had found the food). In the test phase (following a 5-min or 4-h delay), monkeys
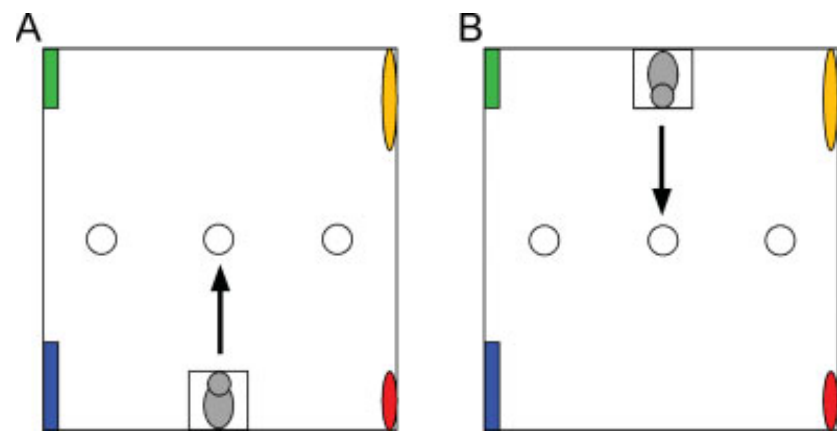

FIGURE 5. Schematic representation of the open-field delayedmatching-to-location task developed by Hampton et al. (2004). During the sample phase, monkeys were allowed to search for food among several potentially rewarded locations and take part of the food reward before being pulled back to the start location. During the test phase, monkeys were allowed to search for the remaining of the food. (A) Monkeys start from the same location in the room during the sample and test phases ("same" trials). (B) Monkey start from a different, opposite location in the room during sample and test phases ("switch" trials). Note that in both conditions, an egocentric frame of reference enables the encoding of and memory for the location of the reward at the center of the array. See main text for a detailed description of the procedure. [Color figure can be viewed in the online issue, which is available at www.interscience.wiley.com.] 
were allowed to search until they found the remaining food located where they had found it originally. Each monkey participated in 25 trials ( 1 trial/day, 5- to 6-days/week).

Hampton et al. showed that the performance of hippocampal-lesioned monkeys did not differ between sample and test phases. Unfortunately, however, although control monkeys performed slightly above chance, they did not exhibit robust match-to-location performance at either $5 \mathrm{~min}$ or $4 \mathrm{~h}$ (NB: This poor performance might be explained by the fact that monkeys were not allowed to search all potentially rewarded locations in the sample phase). Although the lack of a robust performance from the control monkeys makes the results of this experiment difficult to interpret, it is nevertheless clear that because monkeys always began their searches from the same location, both egocentric and allocentric representations of space could be used to encode the location of the reward. The fact that hippocampal-lesioned animals did not perform above chance cannot be taken as evidence that they were incapable of using an egocentric (hippocampus-independent) representation of space to solve the task, but only that egocentric representations are insufficient to sustain spatial memory performance over extended periods of time ( $5 \mathrm{~min}$ in this task), as previously discussed.

Indeed, this hypothesis regarding the time-dependent nature of egocentric representation was further supported by a second experiment, which introduced various delays to a trial-unique match-to-location task. There were four major differences with Experiment 1 including: (1) the addition of shorter delays (1, $2,3,4,5,10,15,20,25$, and $30 \mathrm{~min}$ ), (2) trial-unique configurations of three foraging sites, (3) a clearly visible reward at the target site during the sample phase, and (4) two or three trials per day. As before, however, monkeys always started the task from the same location. For this series of experiments, all five control monkeys performed the task with delays up to 10 min and two control monkeys succeeded with delays of 30 min. Interestingly, although one of the six hippocampallesioned monkeys failed to reach an arbitrary criterion of $81 \%$ accuracy during a pretraining stage with a 30-s delay, four of the monkeys were able to perform the task following a 1-min delay, and one monkey was even able to perform the task following a 5-min delay. Although this experiment did not control for the presence of egocentric and allocentric spatial frames of reference (i.e., both frames of reference were available to solve the task), it demonstrated that hippocampal-lesioned monkeys could succeed at shorter delay intervals (i.e., shorter intervals compared to controls), suggesting that egocentric representations are insufficient to sustain spatial memory performance over extended periods of time.

In Experiments 3 and 4, Hampton et al. attempted to control for the different spatial frames of reference that monkeys could use to solve their task by introducing two different start locations in the test phase (Fig. 5). Monkeys were tested with three foraging sites and a 1-min delay between sample and test phases. Now during the test phase, however, monkeys could either be released to start searching from the same side of the room as they had experienced during the sample phase ("same" trials; Fig. 5A), or they could be released to start searching from the opposite side of the room from which they had experienced during the sample phase ("switch" trials; Fig. 5B). The authors predicted that control monkeys would be able to find the reward in both the same and the switch trials, whereas hippocampal-lesioned monkeys should be able to find the reward during the same, but not the switch trials. Unfortunately, however, the control monkeys' performance did not differ from that of hippocampal-lesioned monkeys during either the same or the switch trials. Performance of both groups of monkeys decreased during switch trials, as compared to same trials, but nevertheless remained above chance level, even for the hippocampal-lesioned monkeys.

If an allocentric, relational representation of space is necessary to solve the task by Hampton et al. in the switch condition, a presumably relational task, then the better than chance performance of the hippocampal-lesioned animals is particularly surprising. However, a closer look at the task indicates that an allocentric, relational representation of space is not necessary to perform above chance (Fig. 5). As before, an egocentric coding of space could enable a subject to perform at above chance levels even in the switch trials if one designates the three foraging sites as left, center, and right. Then, regardless of whether one starts at the same location, or at the opposite (switch) location in the test trial, the site at the center of the configuration can always be encoded and remembered as the center location. Consequently, when the center location is rewarded (i.e., 33\% of the trials), monkeys can use a preserved egocentric spatial representation to remember its location, and can therefore be correct on one-third of the trials. During the other two-thirds of trials (when either the left or the right locations are rewarded), monkeys have a $50 \%$ chance of being correct when starting in the switch condition by simply choosing randomly between left and right (here, monkeys need only to remember "not center" to be correct on $50 \%$ of the trials). Thus, if one calculates the probability for all three types of trials together, monkeys have a $66 \%$ chance of being correct when simply relying on an egocentric representation of space $[(1 / 3 \times 100 \%)+$ $(2 / 3 \times 50 \%)=66 \%$ ]. Interestingly, this is exactly the performance observed for one hippocampal-lesioned monkey ("Sm") in the "switch" condition. Note also that this monkey performed at $100 \%$ correct in the "same" condition, and that it also happened to be the best performer among hippocampallesioned monkeys in Experiments 1 and 2. Moreover, the poor performance of control monkeys on the switch trials (i.e., an average of $45 \%$ correct) may similarly be explained by reliance on an egocentric strategy.

In sum, the experiments by Hampton et al. not only failed to distinguish controls from hippocampal-lesioned monkeys (Experiments 1, 3, and 4) and to preclude monkeys from relying on an egocentric spatial frame of reference (Experiment 4), but also failed to demonstrate that normal monkeys were relying on an allocentric representation of space to solve their open-field task (Experiments 3 and 4). In fact the data suggest that, in contrast to what the authors claim, some control monkeys were indeed relying on an egocentric strategy to solve the 
task. Thus, although their study attempted to address the issue of egocentric versus allocentric spatial representations, it remained inconclusive with respect to the role of the monkey hippocampus in the establishment or use of allocentric, spatial relational representations of the environment.

Hampton et al. (2004) discussed previously reported differences between spatial memory studies based on the use of large-scale versus small-scale environments: "One possible reason for the preponderance of negative findings [for a role of the hippocampus in spatial learning and memory] in nonhuman primates is that the tests used with monkeys differ dramatically in relative spatial scale from those used with rodents and birds. In contrast with the tests with smaller animals, monkeys were not required to navigate through space in a testing arena many times their own body size, but rather responded by reaching out to the test stimuli from a single body position." Although at the end they concluded that the size of the testing environment could not explain the inconsistent results of previous studies, they themselves did not provide a clear explanation. We believe that the only critical factor explaining the discrepancy between the previous studies, as alluded to by Hampton et al., is the fact that monkeys were only able to respond by reaching out to the test stimuli from a single body position. In other words, both normal and hippocampal-lesioned monkeys could rely on egocentric representations of space to guide their behavior, which, as we have just discussed, was also the case in the experiments by Hampton et al. (2004), irrespective of the scale of the environment.

\section{THE OPEN-FIELD FORAGING PARADIGM}

We have recently developed and implemented a novel experimental paradigm to clarify the role of the monkey hippocampus in allocentric, spatial relational learning and memory (Banta Lavenex et al., 2006; Lavenex and Banta Lavenex, 2006; Lavenex et al., 2007b). As discussed previously, we believed that it was imperative to test monkeys in conditions that clearly distinguish between egocentric (viewpoint-dependent) and allocentric (viewpoint-independent) representations of space. Accordingly, our task, a paradigm previously designed to study spatial learning and memory in rodents (Lavenex and Schenk, 1995, 1996, 1997, 1998; Lavenex et al., 1998), explicitly precludes the ability of monkeys to rely on an egocentric strategy to solve the task, as we will describe.

Our task is a foraging task, in which monkeys can freely search for food in an open-field environment (Fig. 6). From within the arena, monkeys have visual access to surrounding environmental cues. They forage for preferred food items (e.g., grapes, marshmallows) that are located in two different arrays of three distinct locations (gray circles in Figs. 6A,B). Monkeys behave freely without any negative reinforcement to shape their behavior. They have $5 \mathrm{~min}$ to find all three rewarded locations, and are free to explore the arena for $30 \mathrm{~s}$ after they have found all three rewards [which reduces the animal's drive to explore the environment before choosing the rewarded locations (Lavenex and Schenk, 1995, 1997, 1998; Lavenex et al., 1998)]. There are two critical features of our task that prevent monkeys from using an egocentric strategy to solve the task. First, within the testing arena, 18 regularly arranged inverted cups serve as potential goal locations (although only six of these locations ever contain food; NB: This paradigm thus does not penalize a win-stay strategy). Second, on each trial, the monkey enters the arena from one of four pseudorandomly chosen entrance points (located at the four corners of the arena). Thus, the combination of multiple goal locations and four entrance points explicitly precludes the ability of monkeys to rely on an egocentric strategy to identify the rewarded locations. Specifically, as illustrated in Figure 6A, the performance of a monkey that employs an egocentric, viewpoint-dependent strategy (e.g., second cup on the left) after entering the arena will not choose potentially rewarded locations (gray circles) more frequently than neverrewarded locations (white circles). In contrast, monkeys that employ an allocentric, spatial relational strategy (viewpoint-independent) will correctly choose potentially rewarded locations (Fig. 6B).

Our experiment includes two different conditions: a local cue condition and a spatial relational condition. In the local cue condition (Fig. 6C), rewarded locations are marked by redand blue-colored cups, so that monkeys can use either local cues or spatial relational information to discriminate the food locations. In the spatial relational condition (Fig. 6D), rewarded locations are covered with the same neutral-colored cups as all other locations (i.e., no local cues mark the food locations), so that monkeys must rely on a spatial relational representation of the environment to discriminate these locations. In other words, monkeys must form and utilize an allocentric representation of space that codes the goal locations in relation to distant environmental cues (Fig. 6B), the very definition of spatial relational memory. Again, the combination of multiple goal locations and four pseudorandomly chosen entrance points precludes the ability of monkeys to rely on an egocentric strategy to identify the rewarded locations (Fig. 6A).

We summarize here the results of one of our studies that investigated the role of the monkey hippocampus in allocentric, spatial relational learning and memory (Banta Lavenex et al., 2006). We compared the individual behavior of six monkeys that had received experimental lesion of the hippocampus in adulthood with that of six monkeys that had undergone sham surgery. We found that, as predicted, all hippocampal-lesioned and all sham-operated monkeys preferentially chose the rewarded locations when they were marked by local cues, that is, red and blue cups (Fig. 6C). In contrast, none of the six hippocampal-lesioned monkeys, as compared to five of six sham-operated control monkeys, preferentially chose the rewarded locations in absence of local cues marking these locations (Fig. 6D). Most interestingly, histological analysis of the brain of the sixth control monkey that was not able to preferentially choose the rewarded locations in the spatial relational condition revealed nonexperimentally induced bilateral damage completely confined to the hippocampus (CA regions). More- 
A

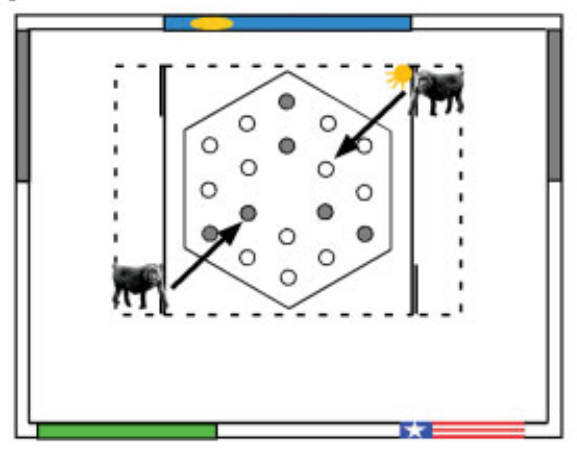

C

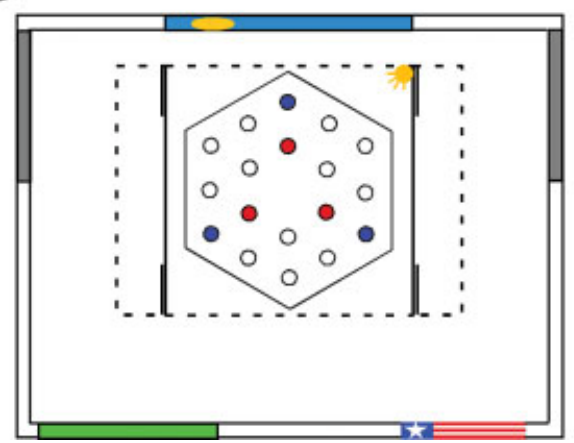

FIGURE 6. Schematic representation of the open-field experimental design developed by Lavenex and Banta Lavenex (2006). Monkeys forage freely for preferred food located in two different arrays of three distinct locations. (A) An egocentric strategy (viewpoint-dependent) does not enable the discrimination of potentially rewarded locations (gray circle) and never-rewarded locations (white circle). (B) An allocentric, spatial relational strategy (viewpoint-independent) enables the discrimination of potentially rewarded locations (gray circles) from never-rewarded locations (white circles). See main text for a detailed description of the pro-
B

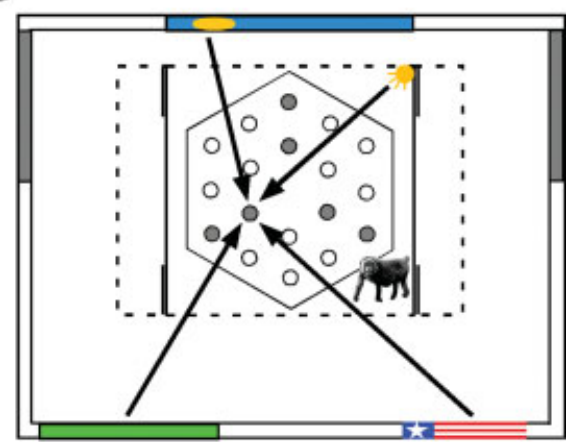

D

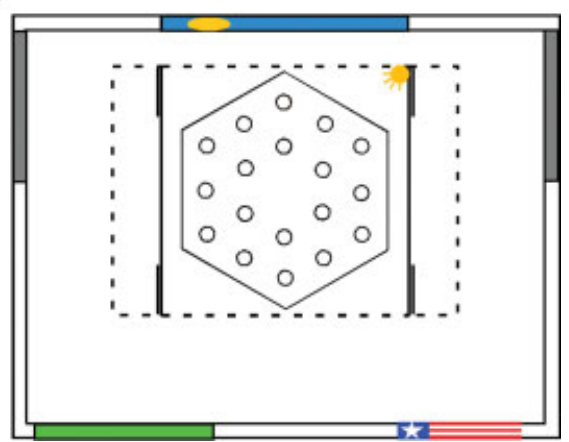

cedure. (C) Local cue condition: red and blue cups mark the potentially rewarded locations. Both normal and hippocampallesioned monkeys are able to identify rewarded locations in this condition. (D) Spatial relational condition: all locations are covered by the same neutral-colored cups. Control animals are capable of identifying rewarded locations, whereas hippocampallesioned animals are incapable of identifying rewarded locations. See main text for a detailed description of the procedure and results. [Color figure can be viewed in the online issue, which is available at www.interscience.wiley.com.] over, the behavior of this monkey in the spatial relational condition (i.e., his location choices) was indistinguishable from that of the experimentally lesioned monkeys (Banta Lavenex et al., 2006).

Why do the results of our experiment investigating the role of the monkey hippocampus in spatial memory differ so drastically from the experiments described above? First, because normal monkeys have a natural ability to use both egocentric and allocentric strategies to solve spatial tasks, we have specifically designed our task to prevent monkeys from relying on egocentric strategies to discriminate the food locations. Our results show that once the information provided by an egocentric frame of reference is made unreliable, hippocampal-lesioned monkeys, who are unable to rely on an allocentric spatial frame of reference, are completely unable to preferentially choose rewarded locations in the absence of local cues marking these locations. Moreover, it is important to recognize that lesioned monkeys were not simply "impaired" in their ability to preferentially choose the rewarded locations (i.e., it is not the case that control monkeys were 90\% correct and lesioned monkeys were $65 \%$ right, but still significantly above chance), but rather their choices did not differ from chance. Restricted hippocampal lesion prevented allocentric, spatial relational processing entirely, in every single lesioned animal. In contrast, all control animals solved the task by making choices that were clearly guided by an allocentric representation of space (Banta Lavenex et al., 2006).

Finally, we would like to note that the effects of selective damage to the hippocampus that we have described pertain only to individuals lesioned in adulthood. In another series of experiments employing this identical open-field task, we found that spatial relational learning in this paradigm persists following neonatal hippocampal lesions in macaque monkeys (Lavenex et al., 2007b). This surprising result thus suggests that early, but not late, hippocampal damage leads to functional brain reorganization that enables spatial information to be acquired through the use of brain regions that normally do not subserve this function. These latter findings further emphasize 
the need for systematic studies of the structural and functional maturation of the primate hippocampal formation in order to gain insight into the specific types of information processing that are subserved by the medial temporal lobe structures at different developmental stages (Lavenex et al., 2007a).

\section{DISCUSSION}

In this commentary, we have explained in detail how previous studies investigating the role of the monkey hippocampus in spatial learning and memory have produced inconsistent results, because the tasks that have been employed can be solved by using egocentric (hippocampal-independent) as well as allocentric (hippocampal-dependent) representations of space. Moreover, we have described how previous findings suggesting that the monkey hippocampus is not critical for spatial memory processes at shorter delays can be explained by the preserved ability of hippocampal-lesioned monkeys to rely on egocentric spatial representations over short time delays. These egocentric representations, however, are not maintained over long periods of time and thus do not sustain performance over long delays. In contrast, we have unequivocally shown, in a task that precludes the use of egocentric strategies, that the monkey hippocampus is critical for the establishment or use of allocentric, spatial relational representations of the environment. In sum, it can be concluded that the role of the hippocampus in spatial learning and memory is conserved among vertebrates, including nonhuman primates.

In light of the arguments developed in this commentary, we propose that the following criteria be adopted by researchers, and considered by readers and reviewers alike when evaluating contributions to the literature. First, use of the words "space" or "spatial" must be qualified. It is clear that not all space is created equal. Studies of spatial memory should thus clearly define the specific kind(s) of spatial representation(s) being tested (e.g., egocentric vs. allocentric), so that the reader can both evaluate the experimental design as well as make predictions about how different brain structures contribute to the elaboration and use of distinct spatial representations.

Second, experiments that aim to assess allocentric, spatial relational memory must be designed in a manner that precludes subjects from using egocentric spatial frames of reference to solve the task. Specifically, any task in which subjects always approach a testing apparatus from the same direction cannot rid the task of its egocentric component, and therefore cannot be considered purely spatial relational. However, just changing the position from which the animal approaches the task is not a fool-proof solution. Egocentric strategies can still be used efficiently from novel start locations, if the task requires animals to discriminate only one to a few goal locations, as we described above.

Finally, although this commentary has been written with an emphasis on current investigations of the role of the hippocampus in spatial relational memory in monkeys, it is obvious that the ideas we have presented are valid for investigations of spatial relational memory in all species, from rodents to humans. However, we believe that by incorporating these suggestions into the current investigations of hippocampal function in nonhuman primates that this domain can finally achieve concensus with findings from other species, and contribute to the advancement of our overall understanding of hippocampal function.

\section{REFERENCES}

Alvarado MC, Wright AA, Bachevalier J. 2002. Object and spatial relational memory in adult rhesus monkeys is impaired by neonatal lesions of the hippocampal formation but not the amygdaloid complex. Hippocampus 12:421-433.

Amaral DG, Lavenex P. 2007. Hippocampal neuroanatomy. In: Andersen P, Morris RGM, Amaral DG, Bliss TV, O'Keefe J, editors. The Hippocampus Book. Oxford: Oxford University Press. pp 37114.

Angeli SJ, Murray EA, Mishkin M. 1993. Hippocampectomized monkeys can remember one place but not two. Neuropsychologia 31:1021-1030.

Bachevalier J, Nemanic S. 2008. Memory for spatial location and object-place associations are differently processed by the hippocampal formation, parahippocampal areas TH/TF and perirhinal cortex. Hippocampus 18:64-80.

Banta Lavenex P, Amaral DG, Lavenex P. 2006. Hippocampal lesion prevents spatial relational learning in adult macaque monkeys. J Neurosci 26:4546-4558.

Beason-Held LL, Rosene DL, Killiany RJ, Moss MB. 1999. Hippocampal formation lesions produce memory impairment in the rhesus monkey. Hippocampus 9:562-574.

Belcher AM, Harrington RA, Malkova L, Mishkin M. 2006. Effects of hippocampal lesions on the monkey's ability to learn large sets of object-place associations. Hippocampus 16:361-367.

Bird CM, Burgess N. 2008. The hippocampus and memory: Insights from spatial processing. Nat Rev Neurosci 9:182-194.

Brown MW, Aggleton JP. 2001. Recognition memory: What are the roles of the perirhinal cortex and hippocampus? Nat Rev Neurosci 2:51-61.

Buckmaster CA, Eichenbaum H, Amaral DG, Suzuki WA, Rapp PR. 2004. Entorhinal cortex lesions disrupt the relational organization of memory in monkeys. J Neurosci 24:9811-9825.

Cohen NJ, Eichenbaum H. 1993. Memory, Amnesia, and the Hippocampal System. Cambridge, Massachusetts: The MIT Press.

Eichenbaum H. 1999. The hippocampus and mechanisms of declarative memory. Behav Brain Res 103:123-133.

Eichenbaum H, Yonelinas AP, Ranganath C. 2007. The medial temporal lobe and recognition memory. Annu Rev Neurosci 30:123-152.

Feigenbaum JD, Rolls ET. 1991. Allocentric and egocentric spatial information processing in the hippocampal formation of the behaving primate. Psychobiology 19:21-40.

Georges-Francois P, Rolls ET, Robertson RG. 1999. Spatial view cells in the primate hippocampus: Allocentric view not head direction or eye position or place. Cereb Cortex 9:197-212.

Hampton RR, Hampstead BM, Murray EA. 2004. Selective hippocampal damage in rhesus monkeys impairs spatial memory in an open-field test. Hippocampus 14:808-818.

Hori E, Tabuchi E, Matsumura N, Tamura R, Eifuku S, Endo S, Nishijo H, Ono T. 2003. Representation of place by monkey hippocampal neurons in real and virtual translocation. Hippocampus 13:190-196. 
Lavenex P, Amaral DG. 2000. Hippocampal-neocortical interaction: A hierarchy of associativity. Hippocampus 10:420-430.

Lavenex P, Banta Lavenex P. 2006. Spatial relational memory in 9month-old macaque monkeys. Learn Mem 13:84-96.

Lavenex P, Schenk F. 1995. Influence of local environmental olfactory cues on place learning in rats. Physiol Behav 58:1059-1066.

Lavenex P, Schenk F. 1996. Integration of olfactory information in a spatial representation enabling accurate arm choice in the radial arm maze. Learn Mem 2:299-319.

Lavenex P, Schenk F. 1997. Olfactory cues potentiate learning of distant visuospatial information. Neurobiol Learn Mem 68:140-153.

Lavenex P, Schenk F. 1998. Olfactory traces and spatial learning in rats. Anim Behav 56:1129-1136.

Lavenex P, Shiflett MW, Lee RK, Jacobs LF. 1998. Spatial versus nonspatial relational learning in free-ranging fox squirrels (Sciurus niger). J Comp Psychol 112:127-136.

Lavenex P, Banta Lavenex P, Amaral DG. 2007a. Postnatal development of the primate hippocampal formation. Dev Neurosci 29:179-192.

Lavenex P, Banta Lavenex P, Amaral DG. 2007b. Spatial relational learning persists following neonatal hippocampal lesions in macaque monkeys. Nat Neurosci 10:234-239.

Malkova L, Mishkin M. 2003. One-trial memory for object-place associations after separate lesions of hippocampus and posterior parahippocampal region in the monkey. J Neurosci 23:1956-1965.

Morris RGM. 2007. Theories of hippocampal function. In: Andersen P, Morris RGM, Amaral DG, Bliss TV, O'Keefe J, editors. The Hippocampus Book. Oxford: Oxford University Press. pp 581-713.

Morris RGM, Garrud P, Rawlins JNP, Okeefe J. 1982. Place navigation impaired in rats with hippocampal-lesions. Nature 297:681-683.

Murray EA, Mishkin M. 1998. Object recognition and location memory in monkeys with excitotoxic lesions of the amygdala and hippocampus. J Neurosci 18:6568-6582.

Nadel L. 1991. The hippocampus and space revisited. Hippocampus $1: 221-229$

Nadel L, Hardt O. 2004. The spatial brain. Neuropsychology 18:473-476.
O'Keefe J. 2007. Hippocampal neurophysiology in the behaving animal. In: Andersen P, Morris RGM, Amaral DG, Bliss TV, O'Keefe J, editors. The Hippocampus Book. Oxford: Oxford University Press. pp 37-114.

O'Keefe J, Dostrovsky J. 1971. The hippocampus as a spatial map. Preliminary evidence from unit activity in the freely-moving rat. Brain Res 34:171-175.

O'Keefe J, Nadel L. 1978. The Hippocampus As a Cognitive Map. Oxford: Clarendon Press.

Ono T, Tamura R, Nakamura K. 1991. The hippocampus and space: Are there "place neurons" in the monkey hippocampus? Hippocampus 1:253-257.

Parkinson JK, Murray EA, Mishkin M. 1988. A selective mnemonic role for the hippocampus in monkeys: Memory for the location of objects. J Neurosci 8:4159-4167.

Rolls ET, O'Mara SM. 1995. View-responsive neurons in the primate hippocampal complex. Hippocampus 5:409-424.

Rolls ET, Xiang JZ. 2006. Spatial view cells in the primate hippocampus, and memory recall. Rev Neurosci 17:175-200.

Rolls ET, Miyashita Y, Cahusac PM, Kesner RP, Niki H, Feigenbaum JD, Bach L. 1989. Hippocampal neurons in the monkey with activity related to the place in which a stimulus is shown. J Neurosci 9:1835-1845.

Schenk F, Grobéty M-C, Lavenex P, Lipp H-P. 1995. Dissociation between basic components of spatial memory in rats. In: Alleva E, Fasolo A, Lipp H-P, Nadel L, Ricceri L, editors. Behavioural Brain Research in Naturalistic and Semi-Naturalistic Settings. NATO ASI series, Series D, Behavioural and Social Sciences. The Netherlands: Kluwer. pp 277-300.

Scoville WB, Milner B. 1957. Loss of recent memory after bilateral hippocampal lesions. J Neurol Neurosurg Psychiatry 20:11-21.

Squire LR, Wixted JT, Clark RE. 2007. Recognition memory and the medial temporal lobe: A new perspective. Nat Rev Neurosci 8:872-883. 